\title{
FINITENESS OF THE LOWER SPECTRUM OF SCHRÖDINGER OPERATORS WITH SINGULAR POTENTIALS
}

\author{
JÖRG DONIG
}

(Communicated by Barbara L. Keyfitz)

Dedicated to Professor Dr. Erhard Meister on the occasion of his sixtieth birthday

\begin{abstract}
We assume that $q: \mathbb{R}^{m} \rightarrow \mathbb{R}(m \geq 3)$ is a measurable function with the property that its negative and positive parts, respectively, beiong to the Kato class $K\left(\mathbb{R}^{m}\right)$ and $K_{\text {loc }}\left(\mathbb{R}^{m}\right)$. We prove a conjecture by B. Simon concerning the finiteness of the lower spectrum of an s.a. realization of the Schrödinger expression $-\Delta+q$ in $L^{2}\left(\mathbb{R}^{m}\right)$ bounded from below.
\end{abstract}

\section{INTRODUCTION}

Let $q: \mathbb{R}^{m} \rightarrow \mathbb{R} \quad(m \geq 3)$ be a measurable function and let $\lambda \in \mathbb{R}$ such that there exist a number $R>0$ and a function $v$ in the complement $E_{R}$ of the closure of the open ball $B_{R}$ (radius $R$, center at the origin) satisfying $v \in C^{2}\left(E_{R}\right)$ and $v>0, q-\lambda=\frac{\Delta v}{v}$ in $E_{R}$. With the use of Gauss's theorem, writing $n$ for the unit outward normal field on $\partial B_{R+1}$, one sees that

$$
\begin{aligned}
\int_{E_{R+1}} & \left\{|\nabla \varphi|^{2}+(q-\lambda)|\varphi|^{2}\right\} \\
= & \int_{E_{R+1}} v^{2}\left|\nabla \frac{\varphi}{v}\right|^{2}-\int_{\partial B_{R+1}} n \frac{\nabla v}{v}|\varphi|^{2} \quad\left(\varphi \in C_{0}^{\infty}\left(\mathbb{R}^{m}\right)\right),
\end{aligned}
$$

of which the estimate

$$
\begin{aligned}
\int_{\mathbb{R}^{m}} & \left\{|\nabla \varphi|^{2}+(q-\lambda)|\varphi|^{2}\right\} \\
& \geq \int_{B_{R+1}}\left\{|\nabla \varphi|^{2}+(q-\lambda)|\varphi|^{2}\right\}-\int_{\partial B_{R+1}} n \frac{\nabla v}{v}|\varphi|^{2} \quad\left(\varphi \in C_{0}^{\infty}\left(\mathbb{R}^{m}\right)\right)
\end{aligned}
$$

is an immediate consequence. Under a further stipulation on $q$, an s.a. operator $T$ in $L^{2}\left(\mathbb{R}^{m}\right)$ which is bounded from below is associated with the left-hand

Received by the editors November 20, 1989 and, in revised form, March 19, 1990; presented to the UAB International Conference on Differential Equations and Mathematical Physics (March 15-21, 1990).

1980 Mathematics Subject Classification (1985 Revision). Primary 35P15; Secondary 47B25, 47F05. 
side of inequality (2). In a seminal work, Piepenbrink [11, Corollary 4.1] proved that the spectrum of $T$ in $(-\infty, \lambda)$ is a finite set. This was accomplished by verifying that the right-hand side of (2) is defined and positive for all elements in the domain of $T$ orthogonal to a finite set in $L^{2}\left(\mathbb{R}^{m}\right)$ composed of eigenfunctions of an appropriate operator with compact resolvent. Allegretto $[2,3,4]$ and B. Simon $[14, \S C 8]$ showed that this result also applies for operators exhibiting a more general principal part or a potential $q$ with a stronger singularity. Assuming that the negative part $q^{-}$of $q$ satisfies

$$
\lim _{r \downarrow 0} \sup _{x \in \mathbb{R}^{m}} \int_{|x-y|<r}|x-y|^{1-m} q^{-} d y=0,
$$

and that the positive part meets a similar local condition, Simon proved the following implication: If there exist a number $R>0$ and a distributional solution $v$ of $(-\Delta+q) v=\lambda v$ in $\mathbb{R}^{m}$ which is positive in $E_{R}$, then $\sigma(T) \cap$ $(-\infty, \lambda)$ is finite (and $\lambda$ is majorized by the infimum of the essential spectrum of $T)$.

Our concern here is to establish the conjecture of Simon [14, p. 518] that the preceding implication still remains true when the negative and positive parts of $q$, respectively, belong to the Kato class $K\left(\mathbb{R}^{m}\right)$ and $K_{\text {loc }}\left(\mathbb{R}^{m}\right)$ (see $\S 1$ ). These weaker stipulations are compatible with a distributional solution $v$ of $(-\Delta+q) v=\lambda v$ in $\mathbb{R}^{m}$ having an unbounded distributional gradient. Apart from confirming inequality (2) in our case, it proves to be difficult to estimate its right-hand side from below. The crucial point is to show that for $\varepsilon>0$ there is a number $\delta>0$ satisfying

$$
\int_{\partial B_{R+1}} n \frac{\nabla v}{v}|\varphi|^{2} \leq \varepsilon \int_{B_{R+1}}|\nabla \varphi|^{2}+\delta \int_{B_{R+1}}|\varphi|^{2} \quad\left(\varphi \in C_{0}^{\infty}\left(\mathbb{R}^{m}\right)\right),
$$

which holds especially when $|\nabla v|$ is bounded on $\partial B_{R+1}$, as guaranteed by Piepenbrink's hypotheses. Evans and Lewis $[5, \S 5]$ have recently noticed that by appealing to the trace theorem and the compactness of the imbedding $\gamma$ : $H^{1}\left(B_{R+1}\right) \rightarrow L^{2}\left(\partial B_{R+1}\right)$, inequality (3) can be verified at most for $\frac{\nabla v}{v} \epsilon$ $L^{p}\left(\partial B_{R+1}\right)$, with $p>1(m=2)$ and $p=m-1 \quad(m \geq 3)$. Since a distributional solution $v$ as above is known merely to meet $(\nabla v)^{2} \in L_{\mathrm{loc}}^{1}\left(\mathbb{R}^{m}\right)$, this observation allows us to prove Simon's conjecture only for $m \leq 3$. We therefore find it appropriate to start out from an equality differing from (1) essentially by the absence of any surface integral (see Proposition 2). Showing that $(\nabla v)^{2} \in K_{\text {loc }}\left(\mathbb{R}^{m}\right)$, we shall then deduce the existence of a number $C>0$ with

$$
\text { (4) } \int_{\mathbb{R}^{m}}\left\{|\nabla \varphi|^{2}+(q-\lambda)|\varphi|^{2}\right\} \geq \frac{1}{2} \int_{B_{R+1}}|\nabla \varphi|^{2}-C \int_{B_{R+1}}|\varphi|^{2} \quad\left(\varphi \in C_{0}^{\infty}\left(\mathbb{R}^{m}\right)\right) \text {. }
$$

Inequality (4) is compared with (2) in proving the desired result by returning to a corollary of the spectral theorem. 
In $\S 1$, after specifying some notation, we shall present our main result (Theorem 1). The full details of the proof will be expounded in $\S 2$. For technical simplifications, the case $m=2$ will be excluded in our considerations.

\section{MAIN ResUlt}

We start with some notation. If $x \in \mathbb{R}^{m}, r>0$, we let

$$
B_{r}(x):=\left\{y\left|y \in \mathbb{R}^{m},\right| x-y \mid<r\right\},
$$

write $B_{r}:=B_{r}(0)$, let $E_{r}$ stand for the complement of $\bar{B}_{r}$, and denote by $\chi_{r}$ the characteristic function of $\bar{B}_{r}$. If $r>0$, we further introduce the set $C_{*}^{\infty}\left(\bar{B}_{r}\right)$ of functions $\varphi: \overline{B_{r}} \rightarrow \mathbb{C}$ with the property that there exists a $\phi \in C_{0}^{\infty}\left(\mathbb{R}^{m}\right)$ with $\varphi=\phi\left\lceil\overline{B_{r}}\right.$.

If $f: \mathbb{R}^{m} \rightarrow \mathbb{R}$ is a measurable function and if $r>0, R>0$, let us put

$$
N_{r}(f):=\sup _{x \in \mathbb{R}^{m}} \int_{B_{r}(x)}|x-y|^{2-m}|f(y)| d y, \quad N_{R, r}(q):=N_{r}\left(\chi_{R} f\right) ;
$$

$f$ is defined to be in the Kato class $K\left(\mathbb{R}^{m}\right)$ and $K_{\text {loc }}\left(\mathbb{R}^{m}\right)$, respectively, provided that

$$
\lim _{r \downarrow 0} N_{r}(f)=0, \quad \lim _{r \downarrow 0} N_{R, r}(f)=0 \quad(R>0) .
$$

It is well known that if, for every $r>0, N_{r}(f)<+\infty$ holds, then there is a number $C=C(m)>0$ such that, for every $r>0$,

$$
\int_{\mathbb{R}^{m}}|f||\varphi|^{2} \leq C N_{r}(f)\left(\int_{\mathbb{R}^{m}}|\nabla \varphi|^{2}+\frac{1}{r^{2}} \int_{\mathbb{R}^{m}}|\varphi|^{2}\right) \quad\left(\varphi \in C_{0}^{1}\left(\mathbb{R}^{m}\right)\right) .
$$

A similar inequality is proved in Schechter [13, Chapter 6, Theorem 9.2].

If $f \in L_{\mathrm{loc}}^{\infty}\left(\mathbb{R}^{m}\right)$, and if $r>0, R>0$, we abbreviate

$$
\sigma_{R, r}(f):=\sup _{\substack{x, y \in B_{R} \\|x-y|<r}}|f(x)-f(y)| .
$$

It is clear that if $f \in C^{0}\left(\mathbb{R}^{m}\right)$, then we have $\inf _{r>0} \sigma_{R, r}(f)=0 \quad(R>0)$.

Finally, let us adopt the following notation. If $f: \mathbb{R}^{m} \rightarrow \mathbb{R}, v: \mathbb{R}^{m} \rightarrow \mathbb{C}$ are measurable functions, and if $\lambda \in \mathbb{R}$, then $v$ is called a distributional solution of the equation $(-\Delta+f) v=\lambda v$ according to $v, f v \in L_{\mathrm{loc}}^{1}\left(\mathbb{R}^{m}\right)$ and

$$
\int_{\mathbb{R}^{m}} v(-\Delta+f-\lambda) \varphi=0 \quad\left(\varphi \in C_{0}^{2}\left(\mathbb{R}^{m}\right)\right) .
$$

After these preparations, we proceed to establish the Schrödinger operator which we shall study. To this end, let $q: \mathbb{R}^{m} \rightarrow \mathbb{R}$ be a measurable function with $q \in$ $L_{\text {loc }}^{1}\left(\mathbb{R}^{m}\right)$, and let $q^{+}$and $q^{-}$be the positive and negative parts, respectively, of $q$, i.e., $q^{+}, q^{-} \geq 0, q=q^{+}-q^{-}$. Let us introduce the Hermitian sesquilinear forms $s, s^{\prime}$, and $t$ on $C_{0}^{\infty}\left(\mathbb{R}^{m}\right)$ by

$$
s(\varphi, \psi):=\int_{\mathbb{R}^{m}}\left(\nabla \varphi \cdot \overline{\nabla \psi}+q^{+} \varphi \bar{\psi}\right), \quad s^{\prime}(\varphi, \psi):=-\int_{\mathbb{R}^{m}} q^{-} \varphi \bar{\psi}
$$


$\left(\varphi, \psi \in C_{0}^{\infty}\left(\mathbb{R}^{m}\right)\right)$, and put $t:=s+s^{\prime}$. In view of our purposes, it is adequate to assume additionally that $q^{-} \in K\left(\mathbb{R}^{m}\right)$ and $q^{+} \in K_{\text {loc }}\left(\mathbb{R}^{m}\right)$. Using (5) with $f$ replaced by $q^{-}$, one can then show that $s$ is closable and bounded from below with lower bound zero, and that $s^{\prime}$ is $s$-bounded from below and from above with lower $s$-bound zero. Accordingly, $t$ is closable and bounded from below. Writing $\bar{s}$ and $\bar{t}$, respectively, for the closure of $s$ and $t$, and letting $H_{s}$ be the completion of $C_{0}^{\infty}\left(\mathbb{R}^{m}\right)$ with respect to the scalar product

$$
\langle\varphi, \psi\rangle_{s}:=s(\varphi, \psi)+\int_{\mathbb{R}^{m}} \varphi \bar{\psi} \quad\left(\varphi, \psi \in C_{0}^{\infty}\left(\mathbb{R}^{m}\right)\right),
$$

we have that $D(\bar{t})=D(\bar{s})=H_{s}$ ( $D$ stands for domain). We now define $T$ as the s.a. operator in $L^{2}\left(\mathbb{R}^{m}\right)$ associated with $\bar{t}$ by the first representation theorem (cf. Kato [9, VI, Theorem 2.1]). It is clear that $D(T) \subset H_{s}$, and that $T$ is bounded from below. If $q \in L_{\text {loc }}^{2}\left(\mathbb{R}^{m}\right)$, and if $T_{0}:=(-\Delta+q) \uparrow C_{0}^{\infty}\left(\mathbb{R}^{m}\right)$, then $T$ is equal to the Friedrichs extension of $T_{0}$.

Now we are in a position to state our main result.

Theorem 1. Let $q^{-} \in K\left(\mathbb{R}^{m}\right)$ and $q^{+} \in K_{\text {loc }}\left(\mathbb{R}^{m}\right)$, and let $\lambda \leq \inf \sigma_{e}(T)$. If there is a number $R>0$ with the property that there exists a distributional solution $v$ of $(-\Delta+q) v=\lambda v$ in $\mathbb{R}^{m}$ with $v>0$ in $E_{R}$, then the set $\sigma(T) \cap(-\infty, \lambda)$ is finite.

Proof. Let $R>0$ as described in the theorem be fixed. We provisionally take for granted that inequality (4) holds true. (This inequality will be verified in the course of proving Lemma 4.) by

First, it is clear that the Hermitian sesquilinear form $a$ on $C_{*}^{\infty}\left(\overline{B_{R+1}}\right)$ defined

$$
a(\varphi, \psi):=\frac{1}{2} \int_{B_{R+1}} \nabla \varphi \cdot \overline{\nabla \psi} \quad\left(\varphi, \psi \in C_{*}^{\infty}\left(\overline{B_{R+1}}\right)\right)
$$

is nonnegative and closable. Since $C_{*}^{\infty}\left(\overline{B_{R+1}}\right)$ is dense in $H^{1}\left(B_{R+1}\right)$, we have $H_{a}=D(\bar{a})=H^{1}\left(B_{R+1}\right)$ (notations similar to the above). Let $A$ be the s.a. operator in $L^{2}\left(B_{R+1}\right)$ associated with $\bar{a}$ according to the first representation theorem. We notice that the form domain $Q(A)$ of $A$ meets $Q(A)=H^{1}\left(B_{R+1}\right)$, as is seen by the second representation theorem (see Kato [9, VI, Theorem 2.23]). Since, for $z \in \rho(A)$, the resolvent $R(z, A)$ is further proved to be compact (see Reed and Simon [12, XIII.14, Corollary 1, p. 258]), we know that the spectrum $\sigma(A)$ is discrete, specifically that there is a sequence $\left(\mu_{n}\right)$ in $\sigma(A)$ and an ONB $\left(\varphi_{n}\right)$ in $D(A)$ of real-valued functions with $\mu_{1}<\mu_{2}<\cdots<\mu_{n} \rightarrow \infty(n \rightarrow \infty)$, and $A \varphi_{n}=\mu_{n} \varphi_{n}(n \in \mathbb{N})$. If $C>0$ is a fixed number satisfying (4), then there is evidently an $n_{0} \in \mathbb{N}$ such that $\mu_{n_{0}} \geq C$. By the minimax principle (see Reed and Simon [12, XIII.1, Theorem XIII.2, p. 78]), defining

$$
M:=\left\{u \mid u \in L^{2}\left(B_{R+1}\right), \int_{B_{R+1}} u \varphi_{k}=0\left(k \in\left\{1, \ldots, n_{0}-1\right\}\right)\right\},
$$


and observing the above representation for $Q(A)$, we obtain

$$
\mu_{n_{0}}=\inf _{0 \neq \varphi \in Q(A) \cap M}\left(\frac{\int_{B_{R+1}}\left|A^{1 / 2} \varphi\right|^{2}}{\int_{B_{R+1}}|\varphi|^{2}}\right)=\inf _{0 \neq \varphi \in H^{1}\left(B_{R+1}\right) \cap M}\left(\frac{\frac{1}{2} \int_{B_{R+1}}|\nabla \varphi|^{2}}{\int_{B_{R+1}}|\varphi|^{2}}\right),
$$

and thus

$$
\frac{1}{2} \int_{B_{R+1}}|\nabla u|^{2} \geq C \int_{B_{R+1}}|u|^{2} \quad\left(u \in H^{1}\left(B_{R+1}\right) \cap M\right) .
$$

From (4) we have

$$
\frac{1}{2} \int_{B_{R+1}}|\nabla u|^{2} \leq \bar{t}(u)+(C-\lambda) \int_{\mathbb{R}^{m}}|u|^{2}<\infty \quad\left(u \in H_{s}\right)
$$

whence $u \in H_{s}$ implies $u \in H^{1}\left(B_{R+1}\right)$, and inequality (4) is extended to $\varphi \in$ $H_{s}$. Writing

$$
\widetilde{M}:=\left\{u \mid u \in L^{2}\left(\mathbb{R}^{m}\right), \int_{B_{R+1}} u \varphi_{k}=0\left(k \in\left\{1, \ldots, n_{0}-1\right\}\right)\right\},
$$

and letting $\lambda$ be as stated in the theorem, we infer from (4) and (6) that

$$
\int_{\mathbb{R}^{m}}(T-\lambda) u \cdot \bar{u} \geq 0 \quad(u \in D(T) \cap \widetilde{M}) .
$$

With the help of (7), the conclusion of our theorem now follows from a general result in Glazman [7, Theorem 12, p. 15] based on the spectral theorem.

\section{Tools}

Our first objective is to draw a useful conclusion from inequality (5).

Lemma 1. If, for every $r>0, R>0, N_{R, r}(f)<+\infty$ holds, then for every $R>0$ there is a number $C=C(m, R)>0$ such that, for every $0<r \leq 1$,

$$
\int_{B_{R}}|f||\varphi|^{2} \leq C N_{R, r}(f)\left(\int_{B_{R}}|\nabla \varphi|^{2}+\frac{1}{r^{2}} \int_{B_{R}}|\varphi|^{2}\right) \quad\left(\varphi \in C_{0}^{\infty}\left(\mathbb{R}^{m}\right)\right) .
$$

Proof. Let $R>0$. By an extension theorem (see, e.g., Lions and Magenes [10, Chapter 1, Theorem 8.1]), we obtain a number $C^{\prime}(R)>0$ with the property that, for every $\psi \in C_{*}^{\infty}\left(\overline{B_{R+1}}\right)$, there exists a $\tilde{\psi} \in C_{0}^{1}\left(\mathbb{R}^{m}\right)$ with $\tilde{\psi} \backslash B_{R}=\psi$ and

$$
\int_{\mathbb{R}^{m}}|\tilde{\psi}|^{2} \leq C^{\prime} \int_{B_{R}}|\psi|^{2}, \quad \int_{\mathbb{R}^{m}}\left(|\nabla \tilde{\psi}|^{2}+|\tilde{\psi}|^{2}\right) \leq C^{\prime} \int_{B_{R}}\left(|\nabla \psi|^{2}+|\psi|^{2}\right) .
$$

Thus, letting $\varphi \in C_{0}^{\infty}\left(\mathbb{R}^{m}\right)$, abbreviating $\varphi_{0}:=\varphi \mid \overline{B_{R}}$, and denoting by $\tilde{\varphi}_{0}$ an extension of $\varphi_{0}$ to $\mathbb{R}^{m}$ as above, then according to (5) and (9), we have, for 
$0<r \leq 1$,

$$
\begin{aligned}
\int_{B_{R}}|f||\varphi|^{2} & =\int_{\mathbb{R}^{m}}\left|\chi_{R} f\right|\left|\tilde{\varphi}_{0}\right|^{2} \\
& \leq C N_{R, r}(f)\left\{\int_{\mathbb{R}^{m}}\left|\nabla \tilde{\varphi}_{0}\right|^{2}+\frac{1}{r^{2}} \int_{\mathbb{R}^{m}}\left|\tilde{\varphi}_{0}\right|^{2}\right\} \\
& \leq C C^{\prime} N_{R, r}(f)\left\{\int_{B_{R}}|\nabla \varphi|^{2}+\frac{1}{r^{2}} \int_{B_{R}}|\varphi|^{2}\right\} .
\end{aligned}
$$

Replacing $C C^{\prime}$ by $C$, we arrive at the asserted inequality.

Our next purpose is to consider some basic properties of distributional solutions to Schrödinger equations. Throughout the following, we always understand $q: \mathbb{R}^{m} \rightarrow \mathbb{R}$ to be a measurable function.

Lemma 2. Let $q \in L_{\mathrm{loc}}^{1}\left(\mathbb{R}^{m}\right)$ and $\lambda \in \mathbb{R}$. If $v$ is a distributional solution of $(-\Delta+q) v=\lambda v$ in $\mathbb{R}^{m}$ with $v \in L_{\mathrm{loc}}^{\infty}\left(\mathbb{R}^{m}\right)$, and if $\nabla v$ and $\Delta v$ are, respectively, the distributional gradient and Laplacian, then we have $\nabla v \in L_{\mathrm{loc}}^{2}\left(\mathbb{R}^{m}\right), \Delta v \in$ $L_{\text {loc }}^{1}\left(\mathbb{R}^{m}\right)$. If, in addition, $q \in K_{\mathrm{loc}}\left(\mathbb{R}^{m}\right)$, then $v \in C^{0}\left(\mathbb{R}^{m}\right)$.

The first implication is an easily verified result (see also Simon [14, Proposition B.3.2, Lemma C.2.1]). As for the second, we refer to Aizenman and Simon [1, Theorem 1.5].

We show that $(\nabla v)^{2} \in K_{\mathrm{loc}}\left(\mathbb{R}^{m}\right)$, but we first state a preparative result.

Proposition 1. Let $q \in K_{\mathrm{loc}}\left(\mathbb{R}^{m}\right)$ and $\lambda \in \mathbb{R}$. If $v$ is a real-valued distributional solution of $(-\Delta+q) v=\lambda v$ in $\mathbb{R}^{m}$, and if $x \in \mathbb{R}^{m}, r>0$, then there is a uniquely determined harmonic function $h$ in $B_{r}(x)$ such that

$$
v(y)=\frac{-1}{(m-2) \omega_{m}} \int_{B_{r}(x)}|y-z|^{2-m}[q(z)-\lambda] v(z) d z+h(y) \quad\left(y \in B_{r}(x)\right) .
$$

$\left(\omega_{m}\right.$ is the surface area of the unit sphere in $\mathbb{R}^{m}$.)

Proposition 1 can be confirmed by working with regularizations of $v$ and $q v$ (cf. Gilbarg and Trudinger [6, Chapter 7.2]), applying the corresponding result for smooth functions and subsequently using a limiting process. We dispense with carrying out the details.

Lemma 3. Let $q \in K_{\mathrm{loc}}\left(\mathbb{R}^{m}\right)$ and $\lambda \in \mathbb{R}$. If $v$ is a real-valued distributional solution of $(-\Delta+q) v=\lambda v$ in $\mathbb{R}^{m}$, then there is a number $C=C(m)>0$ with the property that, for $x \in \mathbb{R}^{m}, 0<r<\frac{1}{4}$, and $R>0$. We have

$$
C \int_{B_{r}(x) \cap B_{R}}|x-y|^{2-m}(\nabla v(y))^{2} d y \leq\left(N_{R+1,5 r}((q-\lambda) v)\right)^{2}+\left(\sigma_{R+1, r}(v)\right)^{2} .
$$

Proof. Without loss of generality, let us assume that $\lambda=0$, and let $v$ be as above. Let $x \in \mathbb{R}^{m}, 0<r<\frac{1}{4}, R>0$ be fixed; furthermore, put

$$
u(y):=\frac{-1}{(m-2) \omega_{m}} \int_{B_{3 r}(x)}|y-z|^{2-m}(q v)(z) d z \quad\left(y \in B_{3 r}(x)\right),
$$


and let $h$ be the harmonic function in $B_{3 r}(x)$ with

$$
v(y)=u(y)+h(y) \quad\left(y \in B_{3 r}(x)\right)
$$

according to Proposition 1. We first prove that there is a number $C=C(m)>0$ with

$$
\int_{B_{r}(x) \cap B_{R}}|x-y|^{2-m}(\nabla u(y))^{2} d y \leq C\left(N_{R+1,4 r}(q v)\right)^{2} .
$$

To begin with, we note that

$$
\left.\nabla u(y)=\frac{1}{\omega_{m}} \int_{B_{3 r}(x)}(y-z)|y-z|^{-m}(q v)(z) d z \quad \text { (a.a. } y \in B_{3 r}(x)\right) .
$$

We further observe that, for $\delta>0$, the function

$$
f_{\delta}(y):=\int_{B_{3 r}(x)}|y-z|^{\delta-m}|(q v)(z)| d z \quad\left(\text { a.a. } y \in B_{3 r}(x)\right)
$$

is in $L^{1}\left(B_{3 r}(x)\right)$. In particular, we have $|\nabla u| \in L^{1}\left(B_{3 r}(x)\right)$. But, since by Lemma $2 \nabla v \in L_{\text {loc }}^{2}\left(\mathbb{R}^{m}\right)$, from (12) we obtain $\nabla u \in L^{2}\left(B_{r}(x)\right)$.

We now set about estimating several integrals. First, we at once have, for $\delta>0$,

$$
\begin{aligned}
\int_{B_{3 r}(x)} & |y-z|^{1+\delta / 2-m}|q v| d z \\
\quad \leq & \left.\int_{B_{3 r}(x)}|y-z|^{1-m}|q v| d z \quad \text { (a.a. } y \in B_{3 r}(x)\right) .
\end{aligned}
$$

Further, considering a.a. $y \in B_{r}(x) \cap B_{R}$,

$$
\begin{aligned}
& \left(\int_{B_{3 r}(x)}|y-z|^{1+\delta / 2-m}|q v| d z\right)^{2} \\
& \quad \leq \int_{B_{4 r}(y) \cap B_{R+1}}|y-z|^{2-m}|q v| d z \cdot \int_{B_{3 r}(x) \cap B_{R+1}}|y-z|^{\delta-m}|q v| d z \\
& \quad \leq N_{R+1,4 r}(q v) \int_{B_{3 r}(x) \cap B_{R+1}}|y-z|^{\delta-m}|q v| d z .
\end{aligned}
$$

Next, referring to Hellwig [8, IV, $\S 4.1$, Hilfssatz 1], we obtain a number $C=$ $C(m)$ such that, for $0<\delta<1$,

$$
\int_{\mathbb{R}^{m}}|x-y|^{2-m}|y-z|^{\delta-m} d y \leq C|x-z|^{2+\delta-m} \quad\left(z \in \mathbb{R}^{m}, z \neq x\right) .
$$

We now use (15), apply the theorems of Tonelli and Fubini, and subsequently 
take account of (16), to conclude, for $0<\delta<1$, that

$$
\begin{aligned}
\int_{B_{r}(x) \cap B_{R}}|x-y|^{2-m}\left(\int_{B_{3 r}(x)}|y-z|^{1+\delta / 2-m}|q v| d z\right)^{2} d y \\
\quad \leq N_{R+1,4 r}(q v) \int_{B_{r}(x) \cap B_{R}}|x-y|^{2-m}\left(\int_{B_{3 r}(x) \cap B_{R+1}}|y-z|^{\delta-m}|q v| d z\right) d y \\
\quad \leq N_{R+1,4 r}(q v) \int_{B_{3 r}(x) \cap B_{R+1}}|q v(z)|\left(\int_{\mathbb{R}^{m}}|x-y|^{2-m}|y-z|^{\delta-m} d y\right) d z \\
\quad \leq C \cdot N_{R+1,4 r}(q v) \int_{B_{3 r}(x) \cap B_{R+1}}|x-z|^{2-m}|q v| d z \\
\quad \leq C \cdot\left(N_{R+1,4 r}(q v)\right)^{2} .
\end{aligned}
$$

By virtue of the theorem of B. Levi, noticing that, for $0<|y-z|<1$, obviously $|y-z|^{\delta} \uparrow 1(\delta \downarrow 0)$, we gather from (14) that

$$
\begin{aligned}
\int_{B_{3 r}(x)} & |y-z|^{1-m}|q v| d z \\
\quad= & \left.\lim _{\delta \downarrow 0} \int_{B_{3 r}(x)}|y-z|^{1+\delta / 2-m}|q v| d z \quad \text { (a.a. } y \in B_{r}(x)\right),
\end{aligned}
$$

and further conclude from (17) and (18) that

$$
\begin{aligned}
& \int_{B_{r}(x) \cap B_{R}}|x-y|^{2-m}\left(\int_{B_{3 r}(x)}|y-z|^{1-m}|q v| d z\right)^{2} d y \\
& \quad=\lim _{\delta \downarrow 0} \int_{B_{r}(x) \cap B_{R}}|x-y|^{2-m}\left(\int_{B_{3 r}(x)}|y-z|^{1+\delta / 2-m}|q v| d z\right)^{2} d y \\
& \quad \leq C \cdot\left(N_{R+1,4 r}(q v)\right)^{2},
\end{aligned}
$$

of which estimate (13) is an immediate consequence.

Second, we prove the existence of a number $C=C(m)>0$ satisfying

$$
\int_{B_{r}(x) \cap B_{R}}|x-y|^{2-m}|\nabla h|^{2} d y \leq C\left(\sigma_{R+1, r}(v)\right)^{2}+C\left(N_{R+1,5 r}(q v)\right)^{2} .
$$

First of all, it is clear that $h \in C^{\infty}\left(B_{3 r}(x)\right)$, and that each component of $\nabla h$ is again harmonic. If $y \in \overline{B_{r}(x)}$, and if $n$ is the continuous unit outward normal field on $\partial B_{r}(y)$, then by the mean-value property of $\nabla h$ and Gauss's theorem, we obtain from (12)

$$
\begin{aligned}
\nabla h(y)= & \frac{m}{\omega_{m} r^{m}} \int_{\partial B_{r}(y)}[v(z)-v(y)] n(z) d o(\dot{z}) \\
& -\frac{m}{\omega_{m} r^{m}} \int_{\partial B_{r}(y)} u(z) n(z) d o(z) .
\end{aligned}
$$


It is not difficult to see that, for $y \in B_{R}$,

$$
\int_{\partial B_{r}(y)}|v(z)-v(y)| d o(z) \leq \omega_{m} \sigma_{R+1, r}(v) \cdot r^{m-1} .
$$

Besides, we have, for $y \in B_{r}(x) \cap B_{R}$,

$$
\begin{aligned}
\int_{\partial B_{r}(y)} & |u|^{2} d o(z) \\
& =\int_{\partial B_{r}(y)}\left|\frac{1}{(m-2) \omega_{m}} \int_{B_{3 r}(x)}\right| z-\left.\left.t\right|^{2-m}(q v)(t) d t\right|^{2} d o(z) \\
& \leq \frac{1}{(m-2)^{2} \omega_{m}^{2}} \int_{\partial B_{r}(y)}\left(\int_{B_{5 r}(z) \cap B_{R+1}}|z-t|^{2-m}|q v| d t\right)^{2} d o(z) \\
& \leq\left(N_{R+1,5 r}(q v)\right)^{2} r^{m-1} .
\end{aligned}
$$

Using (20), (21), and (22), we hence obtain

$$
\begin{aligned}
& \int_{B_{r}(x) \cap B_{R}}|x-y|^{2-m}|\nabla h|^{2} d y \\
& \leq \frac{2 m^{2}}{\omega_{m}^{2} r^{2 m}} \int_{B_{r}(x) \cap B_{R}}|x-y|^{2-m}\left(\int_{\partial B_{r}(y)}|v(z)-v(y)| d o(z)\right)^{2} d y \\
&+\frac{2 m^{2}}{\omega_{m} r^{m+1}} \int_{B_{r}(x) \cap B_{R}}|x-y|^{2-m}\left(\int_{\partial B_{r}(y)}|u|^{2} d o(z)\right) d y \\
& \leq 2 m^{2} \omega_{m}\left(\sigma_{R+1, r}(v)\right)^{2}+2 m^{2} \cdot\left(N_{R+1,5 r}(q v)\right)^{2},
\end{aligned}
$$

which implies estimate (19).

Finally, an application of the Cauchy inequality and the use of (13) and (19) lead to the claimed inequality (11).

As an immediate consequence of Lemma 3, we note the essential result:

Corollary 1. Under the hypotheses of Lemma 3 and with the above meaning of $v$, we have $(\nabla v)^{2} \in K_{\text {loc }}\left(\mathbb{R}^{m}\right)$.

The following identity provides our device for verifying inequality (4). It is characterized by the absence of any surface integral, as was promised in the introduction.

Proposition 2. Let $q \in L_{\text {loc }}^{1}\left(\mathbb{R}^{m}\right)$ and $\lambda \in \mathbb{R}$. If $w \in C^{\infty}\left(\mathbb{R}^{m}\right), w>0$, then we have, for $R>0$,

$$
\begin{aligned}
\int_{E_{R+1}} & \left\{|\nabla \varphi|^{2}+(q-\lambda)|\varphi|^{2}\right\} \\
= & \int_{B_{R+1}}\left\{-\frac{\Delta w}{w}|\varphi|^{2}-2 \frac{\nabla w}{w} \operatorname{Re} \varphi \cdot \overline{\nabla \varphi}+\left(\frac{\nabla w}{w}\right)^{2}|\varphi|^{2}\right\} \\
& +\int_{E_{R+1}}\left\{w^{2}\left|\nabla \frac{\varphi}{w}\right|^{2}+\left(q-\lambda-\frac{\Delta w}{w}\right)|\varphi|^{2}\right\} \quad\left(\varphi \in C_{0}^{\infty}\left(\mathbb{R}^{m}\right)\right) .
\end{aligned}
$$


Proof. This follows from the identity

$$
\begin{aligned}
|\nabla \varphi|^{2} & +(q-\lambda)|\varphi|^{2} \\
& =\nabla\left(\frac{\nabla w}{w}|\varphi|^{2}\right)+w^{2}\left|\nabla \frac{\varphi}{w}\right|^{2}+\left(q-\lambda-\frac{\Delta w}{w}\right)|\varphi|^{2} \quad\left(\varphi \in C_{0}^{\infty}\left(\mathbb{R}^{m}\right)\right)
\end{aligned}
$$

and a repeated application of Gauss's theorem to the first term on the right-hand side of the equation.

We turn our attention now to the proof of inequality (4). As a first step, we shall make detailed use of regularizations. If $f \in L_{\text {loc }}^{1}\left(\mathbb{R}^{m}\right)$, and if $\varepsilon>0$, we denote the regularization of $f$ by $f_{\varepsilon}$; i.e., letting $\eta \in C_{0}^{\infty}\left(\mathbb{R}^{m}\right)$ be a fixed nonnegative and spherically symmetric function satisfying $\eta \uparrow \bar{E}_{1}=0, \int \eta=1$ (integration over $\mathbb{R}^{m}$ ), we put

$$
f_{\varepsilon}(x):=\varepsilon^{-m} \int_{\mathbb{R}^{m}} \eta\left(\frac{x-y}{\varepsilon}\right) f(y) d y \quad\left(x \in \mathbb{R}^{m}\right)
$$

We state

Lemma 4. Let $q \in K_{\mathrm{loc}}\left(\mathbb{R}^{m}\right)$ and $\lambda \in \mathbb{R}$. If $R>0$ is a number such that there exists a distributional solution $v$ of $(-\Delta+q) v=\lambda v$ in $\mathbb{R}^{m}$ with $v>0$ in $E_{R}$, then there is a number $C=C(m, R, q, \lambda, v)>0$ satisfying

$$
\int_{\mathbb{R}^{m}}\left\{|\nabla \varphi|^{2}+(q-\lambda)|\varphi|^{2}\right\} \geq \frac{1}{2} \int_{B_{R+1}}|\nabla \varphi|^{2}-C \int_{B_{R+1}}|\varphi|^{2} \quad\left(\varphi \in C_{0}^{\infty}\left(\mathbb{R}^{m}\right)\right) .
$$

Proof. Let $R>0$ be as described above and, without loss of generality, let $v$ be real-valued. We first claim that there is a number $C=C(v)>0$ such that

$$
\begin{aligned}
\int_{E_{R+1}}\left\{|\nabla \varphi|^{2}+(q-\lambda)|\varphi|^{2}\right\} \geq & -C \int_{B_{R+1}}\left(1+|q|+(\nabla v)^{2}\right)|\varphi|^{2} \\
& -2 C \int_{B_{R+1}}(1+|\nabla v|)|\varphi||\nabla \varphi| \quad\left(\varphi \in C_{0}^{\infty}\left(\mathbb{R}^{m}\right)\right) .
\end{aligned}
$$

In order to prove $(24)$, let $\alpha \in C_{0}^{\infty}\left(\mathbb{R}^{m}\right)$ be a function satisfying $\alpha(x)=1$ $\left(x \in \overline{B_{R+1 / 2}}\right), \alpha(x)=0\left(x \in \overline{E_{R+1}}\right)$, as well as $0 \leq \alpha(x) \leq 1 \quad\left(x \in \mathbb{R}^{m}\right)$, and further put $w:=\alpha+(1-\alpha) v$. We obviously have $w>0$ and, applying Lemma 2 to $v$, we infer that $w \in C^{0}\left(\mathbb{R}^{m}\right), \nabla w \in L_{\text {loc }}^{2}\left(\mathbb{R}^{m}\right)$, and $\Delta w \in L_{\text {loc }}^{1}\left(\mathbb{R}^{m}\right)$. It is also clear that, for $\varepsilon>0$, we have (indicating, as above, a regularization by an index $\varepsilon$ ) $w_{\varepsilon}>0, w_{\varepsilon} \in C^{\infty}\left(\mathbb{R}^{m}\right)$. Moreover, writing $\beta:=1-\alpha$, one verifies, for $\varepsilon>0$, that

$$
\begin{aligned}
& \nabla w_{\varepsilon}=(\nabla \alpha)_{\varepsilon}+(\nabla \beta \cdot v)_{\varepsilon}+(\beta \cdot \nabla v)_{\varepsilon} \quad \text { and } \\
& \Delta w_{\varepsilon}=(\Delta \alpha)_{\varepsilon}+(\Delta \beta \cdot v)_{\varepsilon}+(\beta q v)_{\varepsilon}+2(\nabla \beta \cdot \nabla v)_{\varepsilon} .
\end{aligned}
$$

The proofs of (25) and (26) involve no difficulties; they use only the properties of $v$ and a simple lemma from Gilbarg and Trudinger [6, Chapter 7, Lemma 
7.3]. Noting that $\alpha, \nabla \alpha, \Delta \alpha$, and $v$ are bounded on $\overline{B_{R+2}}$, one obtains a number $K=K(v)$ such that, for $0<\varepsilon \leq 1$,

$$
\begin{aligned}
& \left|\nabla w_{\varepsilon}(x)\right| \leq K\left(1+|\nabla v|_{\varepsilon}(x)\right) \quad\left(x \in \overline{B_{R+1}}\right) \quad \text { and } \\
& \left|\Delta w_{\varepsilon}(x)\right| \leq K\left(1+|q|_{\varepsilon}(x)+(\nabla v)_{\varepsilon}^{2}(x)\right) \quad\left(x \in \overline{B_{R+1}}\right) .
\end{aligned}
$$

If $A>0$ is a number with $w(x) \geq A \quad\left(x \in \overline{B_{R+2}}\right)$, then, for $0<\varepsilon \leq 1$, we have $w_{\varepsilon}(x) \geq A \quad\left(x \in \overline{B_{R+1}}\right)$, and this in conjunction with (27) leads to the estimate

$$
2 \int_{B_{R+1}} \frac{\nabla w_{\varepsilon}}{w_{\varepsilon}} \operatorname{Re}(\varphi \cdot \overline{\nabla \varphi}) \leq 2 \frac{K}{A} \int_{B_{R+1}}\left(1+|\nabla v|_{\varepsilon}\right)|\varphi||\nabla \varphi| \quad\left(\varphi \in C_{0}^{\infty}\left(\mathbb{R}^{m}\right)\right)
$$

Similarly, using (28), we obtain, for $0<\varepsilon \leq 1$,

$$
\begin{aligned}
& \int_{B_{R+1}} \frac{\Delta w_{\varepsilon}}{w_{\varepsilon}}|\varphi|^{2} \\
& \quad \leq \frac{K}{A} \int_{B_{R+1}}\left\{1+|q|_{\varepsilon}+(\nabla v)_{\varepsilon}^{2}\right\}|\varphi|^{2} \quad\left(\varphi \in C_{0}^{\infty}\left(\mathbb{R}^{m}\right)\right) .
\end{aligned}
$$

We now employ Proposition 2 and use estimates (29) and (30) to find, for $0<\varepsilon \leq 1$, that

$$
\begin{aligned}
\int_{E_{R+1}} & \left\{|\nabla \varphi|^{2}+(q-\lambda)|\varphi|^{2}\right\} \\
\geq & -\frac{K}{A} \int_{B_{R+1}}\left\{1+|q|_{\varepsilon}+(\nabla v)_{\varepsilon}^{2}\right\}|\varphi|^{2}-2 \frac{K}{A} \int_{B_{R+1}}\left(1+|\nabla v|_{\varepsilon}\right)|\varphi||\nabla \varphi| \\
& -\int_{E_{R+1}} \frac{1}{v_{\varepsilon}}\left|-\Delta v_{\varepsilon}+(q-\lambda) v_{\varepsilon}\right||\varphi|^{2} \quad\left(\varphi \in C_{0}^{\infty}\left(\mathbb{R}^{m}\right)\right) .
\end{aligned}
$$

By letting $\varepsilon \rightarrow 0$, we will show that (31) changes into the stated inequality (24). In fact, if $\varphi \in C_{0}^{\infty}\left(\mathbb{R}^{m}\right)$ is fixed, then there are numbers $r=r(\varphi)>0$ and $A=A(\varphi)>0$ with $\operatorname{supp} \varphi \subset \overline{B_{r}}$ and, for $0<\varepsilon \leq 1, w_{\varepsilon}(x) \geq A\left(x \in \overline{B_{r}}\right)$. As a consequence, we obtain

$$
\begin{gathered}
\int_{E_{R+1}} \frac{1}{v_{\varepsilon}}\left|-\Delta v_{\varepsilon}+(q-\lambda) v_{\varepsilon}\right||\varphi|^{2} \leq \int_{B_{r}} \frac{1}{w_{\varepsilon}}\left|-(q v)_{\varepsilon}+q v_{\varepsilon}\right||\varphi|^{2} \\
\leq \frac{1}{A} \int_{B_{r}}\left|q v-(q v)_{\varepsilon}\right||\varphi|^{2}+\frac{1}{A} \sup _{B_{r}}\left|v-v_{\varepsilon}\right| \int_{B_{r}}|q||\varphi|^{2} .
\end{gathered}
$$

Since $(q v)_{\varepsilon} \rightarrow q v$ in $L_{\text {loc }}^{1}\left(\mathbb{R}^{m}\right)$, and since $v_{\varepsilon} \rightarrow v$ uniformly on every compact set as $\varepsilon \rightarrow 0$, we gather from (32) that

$$
\int_{E_{R+1}} \frac{1}{v_{\varepsilon}}\left|-\Delta v_{\varepsilon}+(q-\lambda) v_{\varepsilon}\right||\varphi|^{2} \rightarrow 0 \quad(\varepsilon \rightarrow 0) .
$$

According to (33) and the fact that $|q|_{\varepsilon} \rightarrow|q|$ and $(\nabla v)_{\varepsilon}^{2} \rightarrow(\nabla v)^{2}$ in $L_{\mathrm{loc}}^{1}\left(\mathbb{R}^{m}\right)$ as $\varepsilon \rightarrow 0$, inequality (24) follows, letting $C$ stand for $K / A$. 
Now, on account of (24), there is a number $C=C(v)>0$ such that, for $0<\theta \leq 1$, we have

$$
\begin{aligned}
\int_{\mathbb{R}^{m}}\left(|\nabla \varphi|^{2}+(q-\lambda)|\varphi|^{2}\right) & \\
\geq & (1-2 C \theta) \int_{B_{R+1}}|\nabla \varphi|^{2}-\frac{2 C}{\theta} \int_{B_{R+1}}|\varphi|^{2}-(1+|\lambda|+C) \int_{B_{R+1}}|q||\varphi|^{2} \\
& \quad-\frac{2 C}{\theta} \int_{B_{R+1}}(\nabla v)^{2}|\varphi|^{2} \quad\left(\varphi \in C_{0}^{\infty}\left(\mathbb{R}^{m}\right)\right) .
\end{aligned}
$$

An appropriate choice of $\theta$ in (34) shows that there is a number $C=C(\lambda, v)>$ 0 with

$$
\begin{aligned}
& \int_{\mathbb{R}^{m}}\left(|\nabla \varphi|^{2}+(q-\lambda)|\varphi|^{2}\right) \geq \frac{3}{4} \int_{B_{R+1}}|\nabla \varphi|^{2}-C \int_{B_{R+1}}|\varphi|^{2} \\
&-C \int_{B_{R+1}}\left(|q|+(\nabla v)^{2}\right)|\varphi|^{2} \\
& \quad\left(\varphi \in C_{0}^{\infty}\left(\mathbb{R}^{m}\right)\right) .
\end{aligned}
$$

Abbreviating $Q:=|q|+(\nabla v)^{2}$, we infer from Corollary 1 that $Q \in K_{\mathrm{loc}}\left(\mathbb{R}^{m}\right)$. In particular, $Q$ meets the assumptions of Lemma 1, whence we obtain a number $C=C(m, R)>0$ such that, for $0<r \leq 1$,

$$
\int_{B_{R+1}} Q|\varphi|^{2} \leq C N_{R+1, r}(Q)\left(\int_{B_{R+1}}|\nabla \varphi|^{2}+\frac{1}{r^{2}} \int_{B_{R+1}}|\varphi|^{2}\right) \quad\left(\varphi \in C_{0}^{\infty}\left(\mathbb{R}^{m}\right)\right) .
$$

By (35) and (36), with an appropriate choice of $r$, we arrive at the stated inequality.

On account of Lemma 4, the proof of Theorem 1 is now complete.

\section{REFERENCES}

1. M. Aizenman and B. Simon, Brownian motion and Harnack inequality for Schrödinger operators, Comm. Pure Appl. Math. 35 (1982), 209-273.

2. W. Allegretto, On the equivalence of two types of oscillation for elliptic operators, Pacific $\mathbf{J}$. Math. 55 (1974), 319-328.

3. __ Spectral estimates and oscillations of singular differential operators, Proc. Amer. Math. Soc. 73 (1979), 51-56.

4. Math. 92 (1981), 15-25.

5. W. D. Evans and R. T. Lewis, Eigenvalues below the essential spectra of singular elliptic operators, Trans. Amer. Math. Soc. 297 (1986), 197-222.

6. D. Gilbarg and N. S. Trudinger, Elliptic partial differential equations of second order, Springer-Verlag, Berlin, 1977.

7. I. M. Glazman, Direct methods of qualitative spectral analysis of singular differential operators, Israel Program for Scientific Translations, Jerusalem, 1965.

8. G. Hellwig, Differentialoperatoren der mathematischen Physik, Springer-Verlag, Berlin, 1964. 
9. T. Kato, Perturbation theory for linear operators, Springer-Verlag, Berlin, 1976.

10. J. L. Lions and E. Magenes, Non-homogeneous boundary value problems and applications, Vol. I, Springer-Verlag, Berlin, 1972.

11. J. Piepenbrink, Finiteness of the lower spectrum of Schrödinger operators, Math. Z. 140 (1974), 29-40.

12. M. Reed and B. Simon, Methods of modern mathematical physics, Vol. IV: Analysis of operators, Academic Press, New York, 1978.

13. M. Schechter, Spectra of partial differential operators, North-Holland, Amsterdam, 1986.

14. B. Simon, Schrödinger semigroups, Bull. Amer. Math. Soc. (N.S.) 7 (1982), 447-526.

Fachbereich Mathematik, Universität Duisburg, D-4100 Duisburg, Federal Republic OF GERMANY 